\title{
International plant molecular biology: a bright future for green science
}

\author{
Sibum Sung, Enamul Huq and Z Jeffrey Chen*
}

\begin{abstract}
A report on the 10th International Congress of Plant Molecular Biology, Jeju, South Korea, October 21-26, 2012.

Keywords development, epigenomics, epigenetics, flowering, gene expression, genomics, hybrids, photobiology, plant biology, polyploids
\end{abstract}

\section{Introduction}

Plant biology is arguably one of the most important fields in biology. Norman Borlaug, the father of the Green Revolution, once said, 'You can't build a peaceful world on empty stomachs and human misery'. Plants not only provide us with food and renewable resources, but also serve as model organisms for genetic and molecular studies relevant to agriculture and human health. At the level of basic science, many discoveries, from hereditary laws, transposons and RNA silencing, to light signaling and circadian rhythms, were first made in plants.

The 10th International Congress of Plant Molecular Biology was held on the beautiful Jeju Island and attracted 1,500 attendees from 59 countries. The meeting included a broad spectrum of talks, ranging from plant genomics to biotechnological applications.

\section{Hormones, environmental signaling and plant development}

The Congress President H Ottoline Leyser (Sainsbury Laboratory, University of Cambridge, UK) gave the opening lecture on the regulation of shoot branching and its plasticity in response to ever-changing environmental conditions. The outgrowth of buds is controlled by the interplay between several phytohormones, including auxin, cytokinin and strigolactones. Auxin is transported

\footnotetext{
*Correspondence: zjchen@austin.utexas.edu

Section of Molecular Cell and Developmental Biology, Center for Computational Biology and Bioinformatics, and Institute for Cellular and Molecular Biology, The University of Texas at Austin, Austin, TX 78712-0159, USA
}

downwards from the shoot tip and inhibits bud outgrowth, whereas cytokinins, which are produced primarily in roots and transported upward to buds, promote bud outgrowth. Strigolactones, which are produced in roots and shoots and transported to buds to repress bud activity, negatively regulate the activity of auxin efflux carriers called PIN proteins, and reduce the transport of auxin from the shoot tip to buds. Leyser concluded that the downward movement of auxin in the main stem inhibits bud activity by preventing auxin transport out of buds, whereas strigolactones dampen auxin transport and enhance competition between buds.

The myriad of phytohormones regulating plant growth and development was highlighted by Joseph Ecker (Salk Institute for Biological Studies, USA), who described the networks of transcription factor regulation and crosstalk in response to the plant hormone ethylene. Ethylene is essential for a variety of plant growth and development processes, including germination, fruit ripening, cell senescence and stress response. Together with Hongwei Guo (Peking University, China) and Jose Alonso (North Carolina State University, USA), they showed that the carboxy-terminal domain of ETHYLENE INSENSITIVE2 (EIN2) is post-translationally cleaved and then translocated into the nucleus. Within the nucleus, this polypeptide fragment stabilizes EIN3, which is a master regulator that promotes ethylene signaling.

Genetic approaches have identified CLAVATA1 (CLV1), CLV2, CLV3 and WUSCHEL (WUS) as being involved in the maintenance of the identity of shoot apical meristem. However, it is not known how the membrane-localized receptor like kinases (CLV1 and CLV2) and the peptide ligand CLV3 regulate WUS. In her plenary lecture on signaling in stem cells and meristems, Jen Sheen (Massachusetts General Hospital, USA) demonstrated that the MAP kinase pathway regulates CLV3 signaling through CLV1/2 kinases to control WUS expression at the shoot apical meristem. David Jackson (Cold Spring Harbor Laboratory, USA) showed that FASCINATED EAR2 (FEA2), a CLV2 ortholog, corresponds to a quantitative trait loci for kernel number in maize. This CLV2/ FEA2-based signaling pathway in the shoot apical meristem is analogous to signaling pathways in other 
organs, such as guard cell signaling and plant innate immune responses.

The complexity of environmental signaling is reflected by circadian rhythms that are regulated by diverse mechanisms, including calcium signaling, nutrient signaling, alternative splicing, and transcriptional and posttranslational processes. Alex Webb (University of Cambridge, UK) used modeling and physiological approaches to study calcium signaling feedback and regulation through clock components. C Robertson McClung (Dartmouth College, USA) shared recent data on iron regulation of the circadian clock in Arabidopsis, which is similar to animal clocks that can be entrained by nutrients and metabolites. McClung also summarized the recent work on the genetic architecture of the circadian clock in Brassica rapa, highlighting that the majority of clock genes are preferentially retained during diploidization, following whole genome triplication in the Brassicaceae. Woe-Yeon Kim (Gyeongsang National University, Korea) shared exciting results on the direct interaction between GIGANTEA and SALT OVERLY SENSITIVE2 (SOS2), and discussed how this interaction provides a molecular framework between circadian clock and salt tolerance in plants. Following on from this, Xiaodong Xu (Hebei Normal University, China) isolated new components regulating the alternative splicing of clock genes. David Somers (Ohio State University, USA) showed the importance of the LOV domain of ZEITLUPE in the regulation of clock and light signaling pathways.

\section{Genomics, genetic diversity and natural variation}

In his plenary lecture, Detlef Weigel (Max Planck Institute, Tubingen, Germany) emphasized the origin and consequences of genetic and epigenetic variation in Arabidopsis. His talk focused on three important points: mutation, selection and speciation. He estimated a mutation rate of one mutation per haploid genome per generation in Arabidopsis. However, he showed that among different ecotypes and species, there are many differentially methylated regions, constituting epialleles and variation within and between related species. This notion was echoed by Danelle Seymour (Max Planck Institute, Tubingen, Germany), who discussed DNA methylation comparisons in three Brassicaceae species. Interestingly, David Baulcombe (University of Cambridge, UK) discussed metastable differentially methylated regions in Arabidopsis ecotypes that are independent of a small RNA biogenesis gene, but are dependent on 5 ' noncoding RNA transcripts.

A large amount of genetic and epigenetic variation in plants is primarily caused by hybridization and whole genome duplication or polyploidization, and it is evident that almost all flowering plants have undergone at least one whole genome duplication in their evolutionary history. Z Jeffrey Chen (University of Texas at Austin, USA) discussed recent work on dissecting genome dosage and hybridization effects on biomass, cell size and seed development. Cell size is affected by ploidy levels, but not by hybridization, while biomass is increased in hybrids but not in polyploids. Seed size, however, is affected by both ploidy and hybridization. Thus, it is possible to create superior hybrid seeds. Mechanistically, maternally inherited small RNAs regulate small RNA-containing transcription factor genes, which in turn directly affect endosperm cellularization and consequently seed size.

\section{Epigenetics and epigenomics in light of plant growth and development}

The theme of epigenetics and epigenomics was discussed throughout the congress. Xiaofeng Cao (Institute of Genetics and Developmental Biology, Beijing, China) illustrated the mechanism of how a subset of transposable elements is controlled through crosstalk between histone modification and DNA methylation in rice. Through domain swap and deletion analysis, Jin Hoe Huh (Seoul National University, Korea) showed that DEMETER-like glycosylases remove 5-methylcytosine in a modular fashion. Daniel Shubert (HeinrichHeine University, Germany) described SWINGER/ CURLY LEAF-INTERACTOR1 (SCI1), a histone binding protein: SCI1 and its homologs coordinate polycomb repressive complex 2 (PRC2) activity either by excluding PRC2 from the phosphorylated substrate or by recruiting PRC2 to the non-phosphorylated substrate. Wen-Hui Shen used a homologous recombination detection betaglucuronidase-reporter system to address the function of histone chaperone families in transcription and DNA repair. Ian Henderson (University of Cambridge, UK) found altered recombination frequency in DNA methylation mutants, indicating that chromatin organization affects genetic recombination.

Both small and long non-coding RNAs play a significant role in the epigenetic regulation of gene expression. Caroline Dean (John Innes Centre, UK) described a group of antisense long non-coding RNAs, known as COOLAIR, originating from flowering locus $\mathrm{C}(F L C)$. Dean proposed a model in which co-transcriptional regulation of antisense non-coding RNAs triggers a series of chromatin modifications to silence $F L C$ in various genetic and environmental conditions. Sibum Sung (University of Texas at Austin, USA) illustrated a mechanism in which another long non-coding RNA from $F L C$, namely, COLDAIR, is required for the enrichment of PRC2 at FLC by vernalization. There are natural variations in the structure of COLDAIR among Arabidopsis accessions, suggesting that variations in a long noncoding RNA structure contribute to diverse vernalization responses in nature. 


\section{Biotechnological applications}

Plant biotechnology is leading the way in applications and will continue to bring innovations into the field. An exciting idea of using epigenetic approaches to genetic manipulation of crops has emerged during the congress. In his plenary lecture, Baulcombe described his research on the evolution of RNA silencing, dating back to his work on virus-induced gene silencing. His lab recently discovered mobile small interfering RNAs by grafting experiments, which led him to propose the use of RNAi for producing a novel type of transgenic crops.

Christopher Voigt (MIT, USA) showed that lessons from the application of synthetic biology to bacterial systems can be used to turn on and off certain controlling elements to rewire biosynthetic pathways for improved productivity in plants. Systems biology approaches have been applied to identify biological pathways that integrate circadian rhythms with nitrogen utilization (Gloria Coruzzi, New York University, USA), as well as cell senescence with plant architecture (Hong-Gil Nam and Daegu Gyeongbuk, Institute of Science and Technology, Korea). Jiayang Li (Chinese Academy of Agricultural Sciences and CAS, Beijing, China) employed rice as a model system to isolate genetic mutants that affect plant architecture. One of the semi-dominant mutants, named Ideal Plant Architecture 1 (Ipa1), displayed substantial improvements in productivity. However, IPA1 is not an ideal plant for growth in different environments or countries. To feed the growing populations in the world, it is essential for plant scientists across the globe to create their own ideal plants, and to transform basic science into new biotechnological applications in agriculture.

\section{Conclusions}

The field of plant biology is making remarkable steps forward in the use of genomics technologies to understand the mechanisms controlling many aspects of development, responses to the environment, hormone signaling, genome evolution, natural variation and epigenetics. It seems that exciting times lie ahead for plant biotechnology that may lead to important applications in agriculture to help feed the growing global population.

\section{Competing interests}

The authors declare that they have no competing interests.

\section{Acknowledgements}

We apologize for excluding many interesting findings that were reported in 10 plenary lectures, 56 concurrent sessions and 5 workshops. We thank PillSoon Song (Jeju National University, Korea), the Chair of the local organizing committee, for providing the relevant information used in this meeting report. Research in the Chen, Huq and Sung labs is supported by grants from the National Institutes of Health and National Science Foundation.

Published: 19 November 2012

doi:10.1186/gb-2012-13-11-323

Cite this article as: Sung S, et al:. International plant molecular biology: a bright future for green science. Genome Biology 2012, 13:323. 